Original Article - Clinical Science

\title{
Epidemiology of uveitis in urban Australia
}

Colby T Hart MBBS, ${ }^{1,3}$ Elena Y Zhu OD, ${ }^{2}$ Carmel Crock BLitt, ${ }^{2,3}$ Sophie L Rogers MEpi ${ }^{1}$ and Lyndell L Lim DMedSci ${ }^{1,2,3}$

${ }^{1}$ Centre for Eye Research Australia, Victoria, Australia; ${ }^{2}$ University of Melbourne, Victoria, Australia; ${ }^{3}$ Royal Victorian Eye and Ear Hospital, Victoria, Australia.

Correspondence: Associate Professor Lyndell Lim, Head of Clinical Trials Research Centre, Centre for Eye Research Australia, 32 Gisborne Street, East Melbourne, Victoria, 3002, Australia

Email: limllp@unimelb.edu.au

Short running title: Epidemiology of uveitis in urban Australia Received 17 December 2018; accepted 27 March 2019 Funding sources / Financial disclosure: None Conflict of interest: A/Prof. Lyndell Lim has received a research grant to her institution at the Centre for Eye Research Australia from Abbvie and Bayer. She also works on an advisory board for Bayer, Abbvie and Allergan.

Keywords: Uveitis, Epidemiology, Posterior uveitis, Anterior uveitis, Panuveitis

This is the author manuscript accepted for publication and has undergone full peer review but has not been through the copyediting, typesetting, pagination and proofreading process, which may lead to differences between this version and the Version of Record. Please cite this article as doi: 10.1111/ceo.13517

This article is protected by copyright. All rights reserved. 


\section{ABSTRACT}

Importance: few prior studies have described the epidemiology of uveitis in the Australian population.

Background: to report the incidence and period prevalence of active uveitis in Melbourne and detail their subtypes and etiologies.

Design: cross-sectional study using retrospective medical record review in a tertiary hospital.

Participants: patients with a coded diagnosis of uveitis who attended the emergency department or specialist ocular immunology clinic at the Royal Victorian Eye and Ear Hospital between November 2014 through October 2015 ( $N=1752)$.

Methods: medical records were reviewed to confirm the date of diagnosis and subtype of uveitis. Incidence and prevalence rates were calculated utilizing estimates of the adult population residing in areas of greater Melbourne with more than 30 ocular-related presentations to the emergency department annually.

Main outcomes and measures: presence and date of onset, anatomical distribution and etiology of uveitis.

Results: during the study period, 734 new cases of uveitis and 502 cases of preexisting uveitis requiring active treatment were confirmed. These figures yielded an incidence of 21.54 (CI 20.03, 23.15) per 100,000 person-years and a period prevalence of 36.27 (CI 34.30, 38.35) per 100,000 persons. The distribution of prevalent uveitis cases was anterior $(75 \%)$, intermediate $(6 \%)$, posterior $(15 \%)$ and panuveitis (4\%). An infectious etiology accounted for $13.4 \%$ of cases, a systemic associated disease for $26.4 \%$ of cases, and no cause was identified in $60.2 \%$ of cases.

Conclusion and relevance: the incidence and prevalence rates of uveitis in urban Australia were lower than recent studies from the United States and Europe. 


\section{INTRODUCTION}

Uveitis is one of the top five causes of blindness in developed countries, accounting for up to $10 \%$ of all cases in America ${ }^{1-4}$. The condition often affects young people of working age, which results in a disproportionately higher cost to society when compared to other leading causes of blindness, such as age-related macular degeneration ${ }^{2}$. Despite this impact, limited information exists regarding the epidemiology of uveitis around the world ${ }^{5}$.

A recent systematic review by Miserocchi et al. showed that the overall worldwide incidence of uveitis is $17-52$ per 100,000 person-years and the prevalence is 38-714 cases per 100,000 people 5 . One Australian study was included in this review, which detailed the aetiology and disease associations of uveitis in Sydney, however this study did not investigate population incidence and prevalence rates ${ }^{6}$. Similarly, a more recently published study from Sydney by by Zagora et al. also focused on the aetiology of uveitis rather than incidence and prevalence values ${ }^{22}$. To our knowledge, no prior studies have been published detailing the incidence and prevalence of uveitis in an urban Australian population.

A large proportion of urban Melbourne is serviced by a single ophthalmology-specific emergency department (ED) based at the Royal Victorian Eye and Ear Hospital (RVEEH). Over 40,000 patients are reviewed annually through this service, with many referrals coming from local doctors, other tertiary hospitals and private ophthalmologists. Patients are also able to self-refer and present to the RVEEH ED independently. In addition to this, a specialist ocular immunology clinic (OIC) is run twice per week at the RVEEH. This clinic oversees the management of chronic and complicated cases of uveitis, and also receives referrals from private ophthalmologists and other tertiary hospitals.

The main objectives of the study were to estimate the population incidence and prevalence rates for uveitis and the different subtypes of uveitis based on the 
Standardization of Uveitis Nomenclature (SUN) working group terminology ${ }^{7}$. In addition, the study aims to describe the aetiology of uveitis in all confirmed cases; and to compare results from this study with other uveitis epidemiology studies.

\section{METHODS}

A retrospective review of medical records from patients presenting to the RVEEH ED or attending the OIC from 1 November 2014 through 30 October 2015 was performed. During this period, patients with a potential diagnosis of uveitis were identified as either having attended the RVEEH ED and received a diagnostic code related to uveitis or having attended the OIC at the RVEEH. All patients who presented to the RVEEH ED were examined by an ophthalmology trainee, and all patients who presented to the OIC had been examined by a uveitis subspecialist. Medical records from these patients were then manually searched by two trained health professionals (one medical doctor and one optometrist) in consultation with a uveitis subspecialist to confirm the diagnosis and type of uveitis. Cases were confirmed as uveitis if they fulfilled the diagnostic criteria outlined by the SUN Working Group ${ }^{7}$ based on examination findings within the medical record. Further detail regarding inclusion and exclusion criteria are outlined in Table 1.

Table 1: Inclusion and exclusion criteria.

Confirmed uveitis cases were classified as prevalent if the patient had been diagnosed with uveitis prior to 1 November 2014 and had documented evidence of active disease during the study period. Incident cases were defined as newly diagnosed cases with active disease during the study period. The date of diagnosis was defined as the date of initial review with documented signs of intraocular inflammation. 
In situations where a patient had multiple diagnoses during the study period, the more severe diagnosis was used to classify that patient for incidence and prevalence calculations. For example, a patient who initially presented with iritis and subsequently developed panuveitis within the study period would be classified as panuveitis rather than anterior uveitis.

\subsection{Statistical analysis}

The incidence and prevalence rates for uveitis with Agresti-Coull 95\% confidence intervals (CI) were estimated using the mid-period population as the denominator. A June 302015 estimate of the resident population in Greater Capital City Statistical Area (GCCSA) of Greater Melbourne was obtained from the Australian Bureau of Statistics (ABS) website ${ }^{8}$. This population denominator was then refined by removing people aged less than 18 years and those residing outside of our defined RVEEH catchment area. The RVEEH catchment area comprised all ABS Statistical Area 2 (SA2) that the ABS defines as part of GCCSA "Greater Melbourne" and from whence there were at least 30 RVEEH ED admissions for ocular reasons in a 12-month period contemporaneous to our study period. The cutoff of 30 or more ED presentations was chosen after assessing the distribution of per-SA2 ED presentations: a threshold of 30 presentations roughly corresponds to the 10th percentile (29.1 presentations) of GCCSA SA2s and the 95th percentile (32.1 presentations) of regional/rural areas. After removing the few metropolitan SA2s with zero population, airports, racecourse and other industrial areas, and the SA2s with fewer than 30-presentations, only $11 \%$ (31/281) of Greater Melbourne SA2s are excluded and this excluded region comprises 2215.5 square kilometres (22.2\% of GCCSA total area) and only 147254 adult residents ( $4.1 \%$ of GCCSA total adult population). The remaining 250 included Greater Melbourne SA2s from whence there were 30 or more ocular ED presentations in a year cover an area of 7774.4 square kilometres and a population of 3408068 adults aged 18 and older. 
Active uveitis cases were included if their residential Australian postcode had more than $10 \%$ of its population residing in an RVEEH-catchment SA2. In short, the population denominator comprised adults who resided in greater-metropolitan Melbourne within areas from whence the RVEEH ED received at least 30 ocular presentations per year. Only active uveitis cases that live within this defined RVEEHcatchment were included in the estimation of incidence and prevalence rates. $\mathrm{A}$ descriptive analysis of the age, gender, anatomical location and systemic diseases was performed on all active cases identified during the study period. Categorical variables were summarized as percentages and compared across treatment groups using Fisher's exact test. Age was summarized as mean (CI) or median (interquartile range; IQR) due to skewed distribution in smaller systemic disease subgroups. Any difference in gender distribution in those with and without a specific systemic disease cause was compared using Fishers exact test. All statistical analyses were undertaken using Stata IC 14.2 for Windows (StataCorp LLC, College Station, TX).

\section{RESULTS}

During the study period, 1752 patients with a diagnostic code related to uveitis were identified for medical record review. Of these patients, 979 were reviewed in the OIC and 773 were managed through the ED. Ten records could not be retrieved and 255 patients (14.6\% of records reviewed) were incorrectly coded as having uveitis. Of the total patients with confirmed uveitis, $1201 / 1487$ (80.8\%) were of working age (aged between 18 and 65 years old, inclusive). Following the application of exclusion criteria, 1236 confirmed cases of uveitis were included for analysis (Figure 1).

Figure 1: Identification of confirmed uveitis cases. Details of the number of cases included following the application of the inclusion and exclusion criteria. Arrows to 
the right indicate patients excluded for the relevant reasons. Downward arrow indicates the number of patients remaining for analysis.

A total of 734 patients (59.4\%) had a new diagnosis of uveitis during the study period. The mid-period adult population residing in areas of greater-metropolitan Melbourne that were well-serviced by the RVEEH was estimated to be $3,408,068$ people. The adult incidence of uveitis in greater-metropolitan Melbourne was therefore 21.54 cases $/ 100,000$ person-years (CI 20.03, 23.15). Of these patients, $375(51.1 \%)$ were male. The average age of patients with newly diagnosed uveitis was 46.2 (CI 45.0, 47.5) years. The majority of incident cases were anterior uveitis $(\mathrm{N}=595 ; 81.1 \%)$, followed by posterior uveitis $(\mathrm{N}=81 ; 11.0 \%)$, intermediate uveitis $(\mathrm{N}=37 ; 5 \%)$ and panuveitis $(\mathrm{N}=21 ; 2.9 \%)$. Within greater-metropolitan Melbourne, the incidence of uveitis based on anatomical location in cases $/ 100,000$ person-years was: anterior 17.46 (CI 16.11, 18.92), intermediate 1.09 (CI 0.78, 1.50), posterior 2.38 (CI 1.91, 2.96) and panuveitis 0.62 (CI 0.40, 0.95) (Table 2).

Our review identified 502 patients (40.6\%) who had a previous diagnosis of uveitis that reactivated during the study period. This yielded a prevalence of uveitis within greater-metropolitan Melbourne of 36.27 cases/100,000 persons (CI 34.30, 38.35) (Table 2). Similar to the incident cases, there was no gender discrepancy for prevalent cases, with 638 being male (51.6\%). Likewise, the average age of patients with reactivated disease was similar, with an average age of 46.5 (CI 45.6, 47.4) years. Anterior uveitis remained the most frequent subtype of uveitis in prevalent cases $(N=920,74.4 \%)$, however intermediate uveitis $(N=72 ; 5.8 \%)$, posterior uveitis $(\mathrm{N}=188 ; 15.2 \%)$ and panuveitis $(\mathrm{N}=56 ; 4.5 \%)$ represented a greater proportion of prevalent cases compared to these subtypes for incident cases $(P=0.001)$. The prevalence of uveitis within Greater Melbourne by anatomical location, reported in cases/100,000 persons was: anterior 26.99 (CI 25.31, 28.80), 
intermediate 2.11 (CI 1.67, 2.66), posterior 5.52 (CI 4.78, 6.37) and panuveitis 1.64 (CI 1.26, 2.14) (Table 2).

The aetiology of uveitis within the patient cohort was infectious in 165 cases (13.4\%) and non-infectious in 1071 cases (86.6\%). Of the non-infectious cases, 327 patients $(30.5 \%)$ had a diagnosed associated systemic disease. Table 3 outlines the complete list of cases of uveitis with an associated systemic disease and their anatomical distribution. There was no significant difference in anatomical subtypes between the presumed idiopathic cases compared to patients with an associated systemic disease $(P=0.136)$.

Forty-three percent (139/327) of cases with a systemic association were HLA-B27 positive and 45 patients (13.8\%) had a pre-existing diagnosis of ankylosing spondylitis; of these, the vast majority were male $(84.4 \%, \mathrm{P}<0.001)$. In contrast, a more even representation was seen in the 139 patients with HLA-B27 positive uveitis without known ankylosing spondylitis, where only $53.2 \%$ were male, $\mathrm{P}=0.719$ ). Sarcoidosis was previously diagnosed in 44 patients (13.5\% of systemic associated cases) with a median age of 52 years (IQR 34.5, 68); of which $28(63.6 \%)$ were female. Sarcoidosis accounted for $13.9 \%(10 / 72)$ of all cases of intermediate uveitis reviewed.

The details of the 165 patients with diagnosed infectious uveitis are outlined in Table 4. It should be noted that conditions that have recently been linked to infectious triggers such as Fuchs uveitis and Eales disease have not been included in this group, as exposure to the causative infections (e.g. Rubella for Fuchs Uveitis) had not been documented or confirmed in our cases. Of our infectious uveitis patients, there was a statistically significantly greater number of posterior uveitis cases compared to patients with idiopathic uveitis (30\%, 49/165 infectious vs $12 \%, 88 / 732$ idiopathic, $\mathrm{P}<0.001)$. Likewise, fewer patients with an infectious aetiology were diagnosed with anterior uveitis $(66.1 \%, 109 / 165)$ compared to patients with idiopathic uveitis $(77.3 \%, 566 / 732, \mathrm{P}<0.001)$. 
Herpes simplex infection was diagnosed in 73 patients and 32 were diagnosed with herpes zoster infection. The majority of patients with herpes simplex infection presented with anterior uveitis $(72 / 73,99 \%)$. The mean age of presentation of herpes simplex infection (57.7 years, CI 53.6-61.8) was not significantly different to age at presentation for herpes zoster (60 years, $C I$ 52.6-67.5) $(P=0.585)$. In contrast, those diagnosed with herpes zoster infection presented with either anterior $(24 / 32,75 \%)$, posterior $(7 / 32,22 \%)$ or panuveitis $(1 / 32,3 \%)$. There were no significant differences by gender $(P>0.05)$. Toxoplasmosis was the next most commonly diagnosed infection, accounting for 29 cases (17.6\%) with a median age at diagnosis in these patients of 34 years (IQR 26, 52). Toxoplasmosis accounted for $14.4 \%(27 / 188)$ of all cases of posterior uveitis that were reviewed.

Table 2: Incidence of uveitis per 100000 person-years and prevalence of uveitis per 100000 persons by location and systemic causes.

Table 3: Distribution of uveitis cases with a systemic association with anatomical distribution, mean age and gender breakdown.

Table 4: Distribution of infectious causes of uveitis with anatomical distribution, mean age and gender breakdown.

\section{DISCUSSION}

This population-based study, the first in an Australian urban population, found that active uveitis is rare within greater-metropolitan Melbourne, with an incidence and prevalence estimated at 21.54/100,000 person-years and 36.27/100,000 persons, respectively. We also showed that the disease affects younger Australians of working 
age ( $80.8 \%$ of confirmed uveitis patients), with no significant gender predilection overall.

Drawing direct comparison between incidence and prevalence values obtained from different studies is difficult due to: variations in study design, era, population cohorts and dissimilarities in healthcare system structures between countries. Our study included patients from the general population of Melbourne who use the public healthcare system, rather than those who utilize private health insurance. When compared to other general population studies, our incidence rate is similar to rates from Finland in the 1980 s (22.6 cases/100,000 person-years) ${ }^{9}$, and lower than rates in Barcelona from 2009-2012 (51.9 cases/100,000 person-years) ${ }^{10}$. Our prevalence rate was lower than those reported in these two studies (68.7 cases $/ 100,000$ persons and 144.8 cases $/ 100,000$ persons, respectively). Our lower prevalence rate may reflect the fact that a number of patients previously diagnosed with uveitis at the RVEEH were subsequently followed up by private ophthalmologists or optometrists in the community and as such further flares would not be captured in our study design. The majority of prevalent cases with subsequent flares likely represent those patients without private health insurance and who therefore have a lower socioeconomic status. We tried to account for this by adjusting our population denominator to only include areas with greater than 30 ocular presentations to the RVEEH ED per year, the assumption being that those areas with fewer than 30 presentations per year are serviced effectively by other public or private ophthalmology services.

We also reported lower incidence and prevalence rates of uveitis compared to studies that included patients based on their medical insurance plan in Northern California in 1998-1999 ${ }^{11}$ (incidence of 52.4 cases/100,000 person-years and prevalence of 115.3 cases $/ 100,000$ persons). The population included in the Northern California publication were a non-randomly selected subset of medical 
insurance members with an under-representation of low-income groups ${ }^{12}$ in contrast to our more general study population.

Other reasons for our lower rates may be differences in case definition methodology and era. Studies from Taiwan between 2000-2008 ${ }^{13}$ (incidence of 122 cases/100,000 person-years) and the United States in $2012^{14}$ (prevalence of 113.5 cases/100,000 persons) included cases identified by diagnostic code only -without medical record review for validation. As prior uveitis studies found that up to $44.9 \%$ of patients coded as uveitis were incorrect upon review of the medical record ${ }^{11}$, this may have led to inflated rates in these studies. Indeed, we found a similarly high rate of miscoding (14.6\%). The effect of the introduction of biologics in the management of the spondyloarthropathies, which account for a large proportion of acute uveitis all uveitis epidemiology studies, may also play a part, given that they have been shown to reduce the rate of anterior uveitis flares in these patients ${ }^{15}$.

The rates of infectious uveitis within our cohort (165/1236 patients, 13.3\%) were significantly lower than those reported in Barcelona $(28.7 \%)^{10}$, India $(31.4 \%)^{16}$ and Italy $(38.7 \%)^{17}$. The lower rates of infectious uveitis in our cohort likely reflects the higher prevalence of the leading causative infectious organisms in these countries compared to Australia. For example, Mercanti et al. found that within a cohort in north-eastern Italy, toxoplasmosis accounted for $17.7 \%$ of all cases of uveitis ${ }^{17}$. Toxoplasmosis infection rates in newborns in Europe are reported as high as 11 cases per 10,000 births $^{18}$, whereas in Australia the reported prevalence is 2.3 cases per 10,000 births $^{19}$. Similarly, Rathinam et al. demonstrated that tuberculosis-related uveitis accounted for $5.6 \%$ of all cases of uveitis within an Indian cohort ${ }^{16}$, where the prevalence of tuberculosis was equivalent to 505 per 100,000 persons $^{20}$, compared to 4.4 per 100,000 persons in Australia ${ }^{21}$.

A strength of this study was the manual confirmation of the diagnosis in all identified cases of uveitis retrieved from central databases. The high rate of uveitis miscoding that we discovered highlights the importance of accurately confirming cases of 
uveitis. A further strength is the high use of dilated fundus examination ( $>99 \%)$ of all our patients by an ophthalmologist, which likely accounts for our higher reported proportion of patients with posterior uveitis than the Northern California study ${ }^{11}$, where a fundus examination was absent from many of their included cases. Another strength was the large quantity of included uveitis cases that were seen at the RVEEH compared to the Northern California ${ }^{11}$ and Barcelona studies ${ }^{10}$. This reflects the large service area and population that was included in our study. A clear limitation is that an unknown number of uveitis cases within Melbourne have not been included in our results due to their management external to the RVEEH. Despite our attempts to adjust for this in our methodology by limiting the population to areas with a large number of RVEEH ED presentations, it is likely that our results still represent an underestimation of the true incidence and prevalence values. Another limitation is the retrospective nature of this study and the inherent biases and errors that are associated with such a study design.

Future studies may be able to more accurately characterize the true epidemiology of uveitis by including patients managed within the community as well as at other tertiary referral centers. However, this will be difficult without a centralized universal medical record system that covers both hospitals and community health care providers such as private ophthalmologists, general practitioners and optometrists. Such a centralized medical record system currently does not exist in Australia.

\subsection{Conclusions}

In this first epidemiology study to report the incidence and prevalence of uveitis in an urban Australian cohort, we found that the incidence of uveitis was lower than those rates reported 15 years prior in Northern California and contemporaneously in Barcelona. There was no significant difference in the rates of uveitis between men and women. The majority of patients in our study were young, and of working age, highlighting the public health impact that uveitis has on the Australian community. 


\section{REFERENCES}

1. Nussenblatt RB. The natural history of uveitis. Int Ophthalmol. 1990;14(5$6): 303-8$.

2. Suttorp-Schulten MS, Rothova A. The possible impact of uveitis in blindness: a literature survey. Br J Ophthalmol. 1996;80(9):844-8.

3. Rothova A, Suttorp-van Schulten MS, Frits Treffers W, Kijlstra A. Causes and frequency of blindness in patients with intraocular inflammatory disease. $\mathrm{Br}]$ Ophthalmol. 1996;80(4):332-6.

4. Bodaghi B, Cassoux N, Wechsler B, et al. Chronic severe uveitis: etiology and visual outcome in 927 patients from a single center. Medicine. 2001;80(4):263-70. 5. Miserocchi E, Fogliato G, Modorati G, Bandello F. Review on the worldwide epidemiology of uveitis. Eur J Ophthalmol. 2013;23(5):705-17.

6. Wakefield D, Dunlop I, McCluskey PJ, Penny R. Uveitis: aetiology and disease associations in an Australian population. Aust N Z J Ophthalmol. 1986;14(3):181-7. 7. Group SoUNW. Standardization of uveitis nomenclature for reporting clinical data. Results of the First International Workshop. Am J Ophthalmol. 2005;140(3):509-16.

8. Australian Bureau of Statistics. 3235.0 - Population by Age and Sex, Regions of Australia. 
http://wwwabsgovau/AUSSTATS/abs@nsf/Lookup/32350Main+Features12015?Open Document. Accessed December 2017.

9. Päivönsalo-Hietanen T, Tuominen J, Vaahtoranta-Lehtonen H, Saari KM. Incidence and prevalence of different uveitis entities in Finland. Acta Ophthalmol Scand. $1997 ; 75(1): 76-81$.

10. Llorenç V, Mesquida M, Sainz de la Maza M, Keller J, Molins B, Espinosa G, et al. Epidemiology of uveitis in a Western urban multiethnic population. The challenge of globalization. Acta ophthalmol. 2015;93(6):561-7.

11. Gritz DC, Wong IG. Incidence and prevalence of uveitis in Northern California: the Northern California epidemiology of uveitis study. Ophthalmology. 2004;111(3):491-500.

12. Gordon NP. Similarity of the adult Kaiser Permanente membership in northern California to the insured and general population in northern California: statistics from the 2007 California Health Interview Survey. Oakland, CA: Kaiser Permanente Division of Research. 2012.

13. Hwang D-K, Chou Y-J, Pu C-Y, Chou P. Epidemiology of uveitis among the Chinese population in Taiwan: a population-based study. Ophthalmology. 2012;119(11):2371-6.

14. Thorne JE, Suhler E, Skup M, Tari S, et al. Prevalence of noninfectious uveitis in the United States: a claims-based analysis. JAMA Ophthalmol. 2016;134(11):1237-45.

15. Braun J, Baraliakos X, Listing J, Sieper J. Decreased incidence of anterior uveitis in patients with ankylosing spondylitis treated with the anti-tumor necrosis factor agents infliximab and etanercept. Arthritis Rheum. 2005;52(8):2447-51. 16. Rathinam S, Namperumalsamy P. Global variation and pattern changes in epidemiology of uveitis. Indian J Ophthalmol. 2007;55(3):173. 
17. Mercanti A, Parolini B, Bonora A, Lequaglie Q, Tomazzoli L. Epidemiology of endogenous uveitis in north-eastern Italy. Analysis of 655 new cases. Acta Ophthalmol Scand. 2001;79(1):64-8.

18. Paul M, Petersen E, Szczapa J. Prevalence of Congenital Toxoplasma gondii Infection among Newborns from the Poznań Region of Poland: Validation of a New Combined Enzyme Immunoassay for Toxoplasma gondii-Specific Immunoglobulin A and Immunoglobulin M Antibodies. J Clin Microbiol. 2001;39(5):1912-6.

19. Walpole I, Hodgen N, Bower C. Congenital toxoplasmosis: a large survey in western Australia. Med J Aust. 1991;154(11):720-4.

20. Chakraborty A. Epidemiology of tuberculosis: current status in India. Indian J Med Res. 2004;120(4):248.

21. Toms C, Stapledon R, Waring J, Douglas P. Tuberculosis notifications in Australia, 2012 and 2013. Commun Dis Intell Q Rep. 2015;39(2):217-35.1.

22. Zagora SL, Symes R, Yeung A, Yates W, Wakefield D, McCluskey PJ. Etiology and clinical features of ocular inflammatory diseases in a tertiary referral centre in Sydney, Australia. Ocular immunology and inflammation. 2017;25(sup1):S107-S14.

\section{TABLES}

Table 1: Inclusion and exclusion criteria.

\section{Inclusion criteria:}

1. Documented evidence of signs of intraocular inflammation on examination findings in the medical record.

\section{Exclusion criteria:}

1. Documented evidence of bacterial or fungal keratitis.

2. Documented evidence of previous intraocular surgery as a cause of inflammation within 3 months prior to presentation. 
3. Documented evidence of a traumatic eye injury as a cause of inflammation in the injured eye within 3 months prior to presentation.

4. Patients aged less than 18 years of age at the commencement of the study period.

5. Patients whose documented residential postcode was outside the reasonable hospital catchment area used to generate the population denominator.

Table 2: Incidence of uveitis per 100000 person-years and prevalence of uveitis per 100000 persons by location and systemic causes.

\begin{tabular}{|c|c|c|c|c|}
\hline & \multicolumn{2}{|c|}{ Incident uveitis } & \multicolumn{2}{|c|}{ Prevalent uveitis } \\
\hline & $\begin{array}{l}\mathrm{N} \\
\text { cases }^{\mathrm{a}}\end{array}$ & $\begin{array}{l}\text { Incidence }(95 \% \mathrm{CI}) \\
\mathrm{b} \text { per } 100000 \\
\text { person-years }\end{array}$ & $\begin{array}{l}\mathrm{N} \\
\text { cases }^{\mathrm{a}}\end{array}$ & $\begin{array}{l}\text { Prevalence } \\
(95 \% \mathrm{CI})^{\mathrm{c}} \text { per } 100 \\
000 \text { persons }\end{array}$ \\
\hline All patients & 734 & $\begin{array}{l}21.54(20.03 \\
23.15)\end{array}$ & 1236 & $\begin{array}{l}36.27(34.30 \\
38.35)\end{array}$ \\
\hline \multicolumn{5}{|l|}{ Anatomical locations } \\
\hline Anterior uveitis & 595 & $\begin{array}{l}17.46(16.11, \\
18.92)\end{array}$ & 920 & $\begin{array}{l}26.99(25.31, \\
28.80)\end{array}$ \\
\hline Intermediate uveitis & 37 & $1.09(0.78,1.50)$ & 72 & $2.11(1.67,2.66)$ \\
\hline Posterior uveitis & 81 & $2.38(1.91,2.96)$ & 188 & $5.52(4.78,6.37)$ \\
\hline Panuveitis & 21 & $0.62(0.4,0.95)$ & 56 & $1.64(1.26,2.14)$ \\
\hline \multicolumn{5}{|l|}{ Causes } \\
\hline $\begin{array}{l}\text { Presumed Idiopathic } \\
\text { uveitis }\end{array}$ & 498 & $\begin{array}{l}14.61(13.38, \\
15.95)\end{array}$ & 744 & $\begin{array}{l}21.83(20.32, \\
23.46)\end{array}$ \\
\hline Systemic association & 147 & $4.31(3.67,5.07)$ & 327 & $9.59(8.61,10.69)$ \\
\hline Infectious causes & 89 & $2.61(2.12,3.22)$ & 165 & $4.84(4.15,5.64)$ \\
\hline
\end{tabular}


a Number of cases of confirmed uveitis from 1 November 2014 to 30 October 2015. Midperiod population residing in Greater Melbourne areas with good coverage by the RVEEH (refined from estimates obtained from the Australian Bureau of Statistics 2015).

${ }^{\mathrm{b}}$ Incidence per 100000 person-years with Agresti-Coull approximations of 95\% confidence intervals for rates.

c Prevalence per 100000 persons with Agresti-Coull approximations of $95 \%$ confidence intervals for rates. 
Table 3: Distribution of uveitis cases with a systemic association with anatomical distribution, mean age and gender breakdown.

\begin{tabular}{|c|c|c|c|c|c|c|c|c|}
\hline \multirow[b]{2}{*}{ Systemic association } & \multirow[b]{2}{*}{$\mathrm{N}$ cases $^{\mathrm{a}}$} & \multicolumn{4}{|c|}{ Location of uveitis, n (\%) } & \multicolumn{3}{|c|}{ Gender } \\
\hline & & Anterior & Intermediate & Posterior & Panuveitis & Median age (IQR) & $\%$ male & $P^{b}$ \\
\hline HLA-B27 positive uveitis & 139 & $\begin{array}{c}134 \\
(96.4)\end{array}$ & $2(1.4)$ & $3(2.2)$ & 0 & $41.0(32.0,49.0)$ & 53.2 & 0.719 \\
\hline Sarcoidosis & 44 & $13(29.6)$ & $10(22.7)$ & $10(22.7)$ & $11(25.0)$ & $52.0(34.5,68.0)$ & 36.4 & 0.046 \\
\hline Ankylosing spondylitis & 45 & $45(100)$ & 0 & 0 & 0 & $43.0(36.0,48.0)$ & 84.4 & $<0.001$ \\
\hline Behcet's disease & 23 & $7(30.4)$ & 0 & $13(56.5)$ & $3(13.0)$ & $37.0(30.0,39.0)$ & 82.6 & 0.003 \\
\hline Vogt-Koyanagi-Harada & 18 & 0 & 0 & $12(66.7)$ & $6(33.3)$ & $36.0(23.0,51.0)$ & 44.4 & 0.637 \\
\hline $\begin{array}{l}\text { Fuchs' Heterochromic } \\
\text { Iridocyclitis }\end{array}$ & 10 & $8(80.0)$ & $1(10)$ & $1(10)$ & 0 & $52.5(40.0,63.0)$ & 90.0 & 0.022 \\
\hline
\end{tabular}

This article is protected by copyright. All rights reserved. 


\begin{tabular}{|c|c|c|c|c|c|c|c|c|}
\hline Juvenile idiopathic arthritis & 8 & $8(100)$ & 0 & 0 & 0 & $23.5(20.5,26.5)$ & 50.0 & 1.0 \\
\hline Eale's disease & 6 & 0 & 0 & $5(83.3)$ & $1(16.7)$ & $31.5(31.0,36.0)$ & 100 & 0.031 \\
\hline Hashimoto's thyroiditis & 6 & $5(83.3)$ & 0 & 0 & $1(16.7)$ & $44.5(42.0,54.0)$ & 16.7 & 0.113 \\
\hline Ulcerative colitis & 5 & $4(80.0)$ & $1(20.0)$ & 0 & 0 & $38.0(34.0,38.0)$ & 60.0 & 1.0 \\
\hline Crohn's disease & 3 & $3(100)$ & 0 & 0 & 0 & $36.0(33.0,68.0)$ & 66.7 & 1.0 \\
\hline Rheumatoid arthritis & 4 & $2(50.0)$ & $1(25.0)$ & $1(25.0)$ & 0 & $60.0(50.0,68.5)$ & 25.0 & 0.359 \\
\hline Reactive arthritis & 4 & $4(100)$ & 0 & 0 & 0 & $34.5(28.0,55.5)$ & 50.0 & 1.0 \\
\hline Multiple sclerosis & 3 & 0 & $2(66.7)$ & $1(33.3)$ & 0 & $50.0(44.0,62.0)$ & 66.7 & 1.0 \\
\hline Sjogren's disease & 2 & $2(100)$ & 0 & 0 & 0 & $33.5(32.0,35.0)$ & 0 & 0.234 \\
\hline Psoriatic arthritis & 2 & $2(100)$ & 0 & 0 & 0 & $32.0(24.0,40.0)$ & 100 & 0.500 \\
\hline
\end{tabular}

This article is protected by copyright. All rights reserved. 


\begin{tabular}{|c|c|c|c|c|c|c|c|c|}
\hline Systemic lupus erythematosus & 2 & 0 & 0 & $2(100)$ & 0 & $48.5(39.0,58.0)$ & 0 & 0.234 \\
\hline Autoimmune retinopathy & 1 & 0 & $1(100)$ & 0 & 0 & 43 & 0 & 0.484 \\
\hline Susac syndrome & 1 & 0 & 0 & $1(100)$ & 0 & 33 & 100 & 1.0 \\
\hline Total & 326 & $\begin{array}{c}237 \\
(72.7)\end{array}$ & $18(5.5)$ & $49(15.0)$ & $22(6.7)$ & $41.0(32.0,52.0)$ & 57.5 & 0.014 \\
\hline
\end{tabular}

a Number of cases does not add to total due to multiple systemic diseases in some subjects. Figures include both incident and preexisting cases of uveitis.

${ }^{\mathrm{b}} p$ values relate to Fishers exact test for gender in those with compared to without that systemic disease. Due to multiple comparison testing these should be interpreted carefully.

Table 4: Distribution of infectious causes of uveitis with anatomical distribution, mean age and gender breakdown.

\begin{tabular}{|c|c|c|c|c|c|c|c|c|}
\hline \multirow[b]{2}{*}{$\begin{array}{l}\text { Infectious } \\
\text { organism }\end{array}$} & \multirow[b]{2}{*}{$\mathrm{N}$ cases $^{\mathrm{a}}$} & \multicolumn{5}{|c|}{ Location of uveitis, n (\%) } & \multicolumn{2}{|c|}{ Gender } \\
\hline & & Anterior & Intermediate & Posterior & Panuveitis & Median age (IQR) & $\%$ male & $p^{b}$ \\
\hline
\end{tabular}




\begin{tabular}{|c|c|c|c|c|c|c|c|c|}
\hline Herpes simplex & 73 & $72(98.6)$ & 0 & $1(1.4)$ & 0 & $60.0(44.0,74.0)$ & 46.6 & 0.400 \\
\hline Herpes Zoster & 32 & $24(75.0)$ & 0 & $7(21.9)$ & $1(3.1)$ & $66.5(42.5,78.0)$ & 46.9 & 0.596 \\
\hline Toxoplasmosis & 29 & $2(6.9)$ & 0 & $27(93.1)$ & 0 & $34.0(26.0,52.0)$ & 65.5 & 0.138 \\
\hline Tuberculosis & 20 & $8(40.0)$ & $3(15.0)$ & $7(35.0)$ & $2(10.0)$ & $35.5(27.0,48.5)$ & 50.0 & 1.0 \\
\hline Syphillis & 5 & $1(20.0)$ & $1(20.0)$ & $3(60.0)$ & 0 & $57.0(54.0,61.0)$ & 80.0 & 0.375 \\
\hline Bartonella & 2 & 0 & 0 & $2(100)$ & 0 & $25.5(23.0,28.0)$ & 0 & 0.234 \\
\hline Hepatitis C & 2 & $1(50.0)$ & 0 & $1(50.0)$ & 0 & $61.0(59.0,63.0)$ & 100 & 0.500 \\
\hline Cytomegalo virus & 1 & $1(100)$ & 0 & 0 & 0 & 37 & 100 & 1.0 \\
\hline Dengue & 1 & 0 & 0 & $1(100)$ & 0 & 42 & 0 & 0.484 \\
\hline Total & 165 & $\begin{array}{c}109 \\
(66.1)\end{array}$ & $4(2.4)$ & $\begin{array}{c}49 \\
(29.7)\end{array}$ & $3(1.8)$ & $52(33,70)$ & 51.5 & 1.000 \\
\hline
\end{tabular}

${ }^{a}$ Number of cases does not add to total due to multiple systemic diseases in some subjects. Figures include both incident and preexisting cases of uveitis.

${ }^{\mathrm{b}} p$ values relate to Fishers exact test for gender in those with compared to without that systemic disease. Due to multiple comparison testing these should be interpreted carefully. 
Figure 1: Identification of confirmed uveitis cases

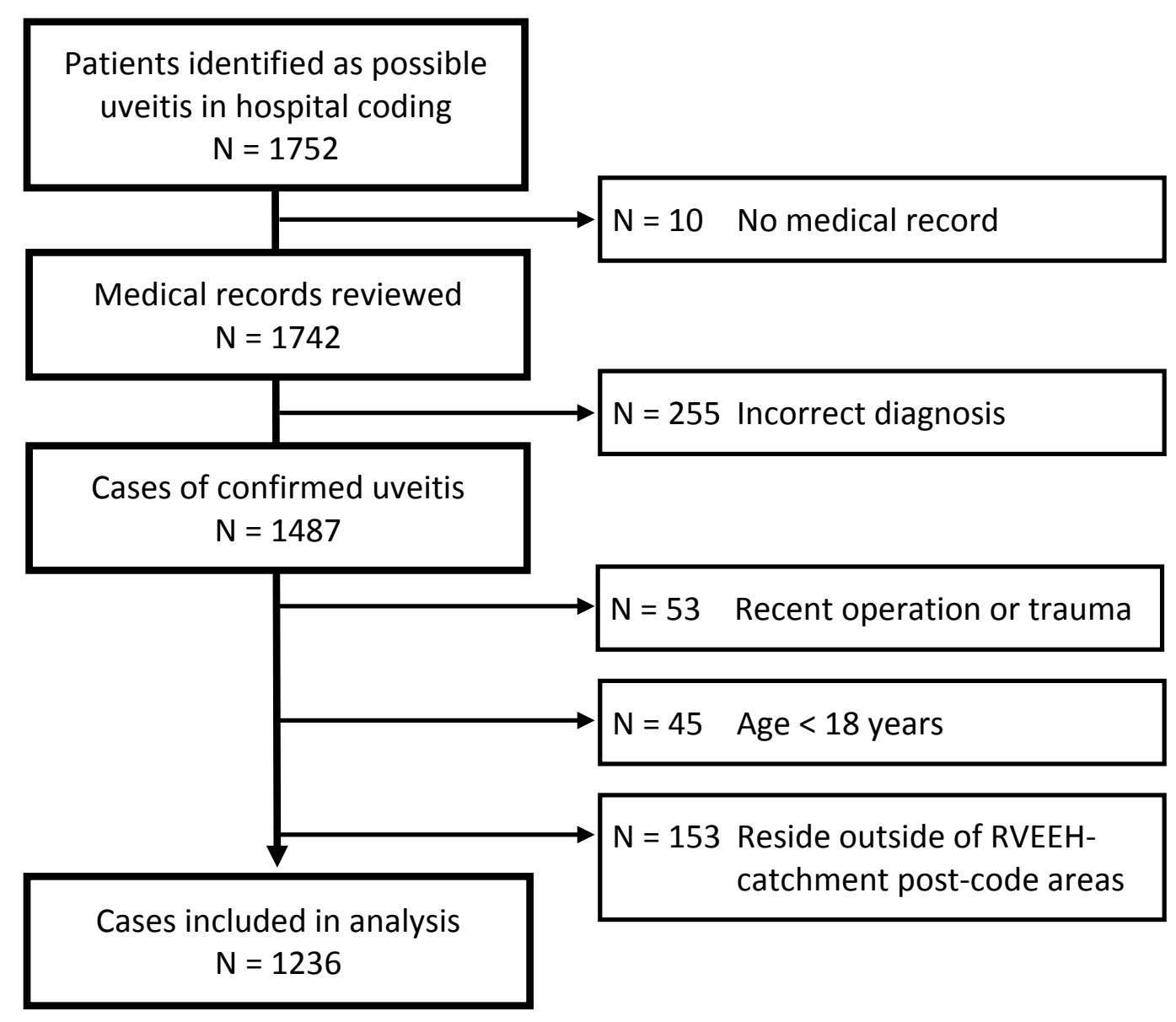

This article is protected by copyright. All rights reserved. 
Details of the number of cases included following the application of the inclusion and exclusion criteria. Arrows to the right indicate patients excluded for the relevant reasons. Downward arrow indicates the number of patients remaining for analysis. 


\section{University Library}

\section{- M M I N E R VA A gateway to Melbourne's research publications}

Minerva Access is the Institutional Repository of The University of Melbourne

Author/s:

Hart, CT;Zhu, EY;Crock, C;Rogers, SL;Lim, LL

Title:

Epidemiology of uveitis in urban Australia

Date:

2019-08-01

Citation:

Hart, C. T., Zhu, E. Y., Crock, C., Rogers, S. L. \& Lim, L. L. (2019). Epidemiology of uveitis in urban Australia. CLINICAL AND EXPERIMENTAL OPHTHALMOLOGY, 47 (6), pp.733-740. https://doi.org/10.1111/ceo.13517.

Persistent Link:

http://hdl.handle.net/11343/285833 